\title{
Spatial-Temporal Characteristics of Primary and Secondary Educational Resources for Relocated Children of Migrant Workers: The Case of Liaoning Province
}

\author{
Dahao Zhang $\mathbb{D}^{1},{ }^{1}$ Chunshan Zhou $\mathbb{D}^{1},{ }^{1}$ and Wenwen $\mathrm{Xu} \mathbb{D}^{2,3}$ \\ ${ }^{1}$ School of Geography and Planning, Sun Yat-sen University, Guangzhou 510275, China \\ ${ }^{2}$ Department of Security, Liaoning Police College, Dalian 116036, China \\ ${ }^{3}$ School of Education, Liaoning Normal University, Dalian 116029, China
}

Correspondence should be addressed to Chunshan Zhou; zhoucs@mail.sysu.edu.cn and Wenwen Xu; xww20202020@163.com

Received 17 April 2020; Revised 5 June 2020; Accepted 20 June 2020; Published 28 July 2020

Guest Editor: Jianhong (Cecilia) Xia

Copyright ( 2020 Dahao Zhang et al. This is an open access article distributed under the Creative Commons Attribution License, which permits unrestricted use, distribution, and reproduction in any medium, provided the original work is properly cited.

Appropriate spatial distribution of primary and secondary schools for relocated children of migrant workers (RCMW) is extremely valuable to ensure the fair education of special groups and improve the quality of their education. This study used concentration analysis, kernel density estimation (KDE), and coefficient of variation (CV) to measure the spatial agglomeration of these children and their primary and secondary schools in the districts and counties of Liaoning Province from 2008 to 2017 based on government surveys. The results showed that the number of RCMW children in primary and secondary schools increased during the research period and the growth rate of children in primary schools was higher than that of children in junior high schools. The number of primary and secondary schools has changed very little, while the number of secondary schools has dropped sharply. The spatial distribution of RCMW and that of their primary and secondary schools were characterized by a "dual-core" distribution and a three-level (county, city, and provincial capital) gradient aggregation. In addition, kernel density evolved from a balanced distribution to polar nucleus-shaped, clustered, and discretized distributions, respectively. Specifically, the "large" and "super-large" schools were mainly concentrated in cities, showing a clustered pattern. "Medium-sized" and "small" schools were mainly distributed in counties, presenting a discretized pattern. In addition, when the CV of the number of RCMW increased, the imbalanced distribution of schools became more significant. Furthermore, the spatial differentiation of primary and secondary schools for RCMW appeared to be affected by economic development, population size, and related policies in the region.

\section{Introduction}

Since the implementation of the reform and opening up policy (economic reform), China's economic development has gradually transitioned from high-speed to high-quality growth. The development of "new-type" urbanization has accelerated, leading to the migration of a large amount of surplus labor from rural to urban areas [1-3]. As a result, the children of migrant workers relocated with their parents and were sent to schools in the corresponding urban areas. Relocated children of migrant workers (RCMW) are children of elementary school age or adolescents who leave the place where the rural household registration is recorded and whose parents move to urban areas where they are required, to receive compulsory education. As of 2018, the total number of RCMW in primary and secondary schools was 13.671 million (10.136 million in primary schools and 3.5354 million in junior high schools). Appropriate spatial distribution of schools not only enhances the accessibility of educational resources in a given region for these children, but also ensures their right to education.

Since the 19th National Congress of the Communist Party of China, the state has prioritized the integrated development of urban and rural education. The "Implementation Plan to Accelerate Education Modernization (2018-2022)," which was jointly issued by the General Office 
of the CPC Central Committee and the General Office of the State Council, asserted that special groups' right to education should be guaranteed and that primary and secondary education of RCMW should be incorporated into urban development planning. The report of the 13th National People's Congress emphasized the need for deepening education reform and ensuring the education of RCMW. In the context of new-type urbanization, the imbalanced development of primary and secondary educational resources for RCMW has become increasingly prominent; specifically, the imbalance was among regions, urban and rural areas, and schools. Thus, it is particularly important to investigate the spatial distribution of schools for these children at the county level.

Research on education-related spatial issues has mainly focused on school-selection decision models [4-6], redistribution of educational resources [7], fairness of educational space [8-10], selection of school location [11-14], spatial relationships between schools and transportation facilities $[15,16]$, problems of separation between schools and resident areas [17-19], school quality [20], and the spatial distribution of students with health conditions in schools [21].

Given the pronounced development of new-type urbanization in China, the number of RCMW has increased [22-25]. Therefore, recent studies have considered the primary and secondary education of these children, such as the spatial distribution of primary and secondary schools [26-28], the correlation between spatial distribution of schools and housing prices [29], the impact of spatial relationships between schools and parents' workplaces on how children are transported to and from school [30], and fairness and accessibility in terms of distribution of school resources [31-33].

In sum, existing studies on spatial characteristics of primary and secondary schools have mainly focused on the following: (1) school-selection decisions and location of schools [34-36]; (2) reallocation of educational resources and spatial distribution of schools [37, 38]; (3) spatial accessibility to educational resources [39-42]; and (4) correlation analysis between spatial distribution of educational resources and social factors, such as social behavior. Missing from the research is the spatial-temporal evolution and balance of schools from the perspective of RCMW against the background of new-type urbanization [43].

As an important industrial province in China, Liaoning Province is undergoing rapid urbanization. The rate of urbanization has grown from $60.05 \%$ in 2008 to $67.37 \%$ in 2017 (average annual growth rate $=0.73 \%$ ). In this sense, Liaoning is representative of Chinese industrial provinces. Thus, this study used Liaoning as the research area and utilized a Geographic Information System (GIS) to conduct a dynamic analysis of the spatial-temporal evolution of primary and secondary schools for RCMW from 2008 to 2017. The purpose of the study was to explore the spatial distribution of these schools in terms of school size, service coverage, concentration, and spatial density at different times to provide a valuable reference for the rational distribution and balanced development of these schools in Liaoning.

\section{Methodology and Research Data}

2.1. Study Area. Liaoning is located in the south of Northeast China, bordering the Bohai Sea and the Yellow Sea; it is separated from North Korea in the east by the Yalu River and from South Korea and Japan by the sea. Liaoning is the only province of the northeastern provinces that is bordered by both land and sea. Therefore, it serves as the main gateway for Northeast China's foreign trade. Liaoning is composed of 14 prefecture-level cities, such as Shenyang, Dalian, Anshan, Fushun City, and Benxi City, and 100 counties (county-level cities), with a total area of approximately 148 thousand kilometers (Figure 1).

2.2. Data Sources. The data used in this study included digital elevation model (DEM) data of $30 \mathrm{~m}$ resolution and coordinates of primary and secondary schools in Liaoning from 2008 to 2017. The DEM data were extracted from the Geospatial Data Cloud. Scrapy was used to extract the coordinates of the schools from Google Earth based on their address. ArcGIS 10.2 was then employed to process the extracted data and construct the spatial distribution map of these schools (Figure 2). The addresses of the schools as well as the number and gender of RCMW were obtained from information statistics collected by the Liaoning Provincial Government. The specific data sources and descriptions are shown in Table 1.

\subsection{Methodology}

2.3.1. Concentration Index. During the process of urbanization, a large number of migrant workers have gradually migrated to towns and counties or from towns and counties to cities, and their children were also transferred from schools in rural areas to those in cities and counties. Due to such continuing concentration, the present authors used the concentration index to analyze the change. Suppose that a prefecture-level city $i$ has $n$ primary and secondary schools, $j_{k}$ symbolizes the number of students in the $k$ th school, the $z$ th school is a city-level school, and the number of students in the school is expressed as $j_{z}$. Thus, the concentration ratio $\left(\mathrm{CR}_{z}\right)(\%)$ of $i$ can be expressed as follows:

$$
\mathrm{CR}_{z}=\frac{j_{z}}{\sum_{k=1}^{n} j_{k}} \times 100
$$

In addition to the main urban area, some prefecture-level cities also have high-tech and development zones. Therefore, in addition to the primary and middle schools in the main urban area, there are schools in the new zones (such as $z, m$, and $f$ ). Therefore, the concentration ratio $\left(\mathrm{CR}_{x}\right)(\%)$ can be calculated as follows:

$$
\mathrm{CR}_{x}=\left\{\frac{j_{z}}{\sum_{k=1}^{n} j_{k}}+\frac{j_{m}}{\sum_{k=1}^{n} j_{k}}+\frac{j_{f}}{\sum_{k=1}^{n} j_{k}}+\cdots\right\} \times 100 .
$$

Suppose a county (or county-level city) has $e$ schools that are located in the built-up area of the county, and the number of students in the $i$ th school is expressed as $j_{c_{i}}$. In addition, there are $f$ schools that are located outside of the 


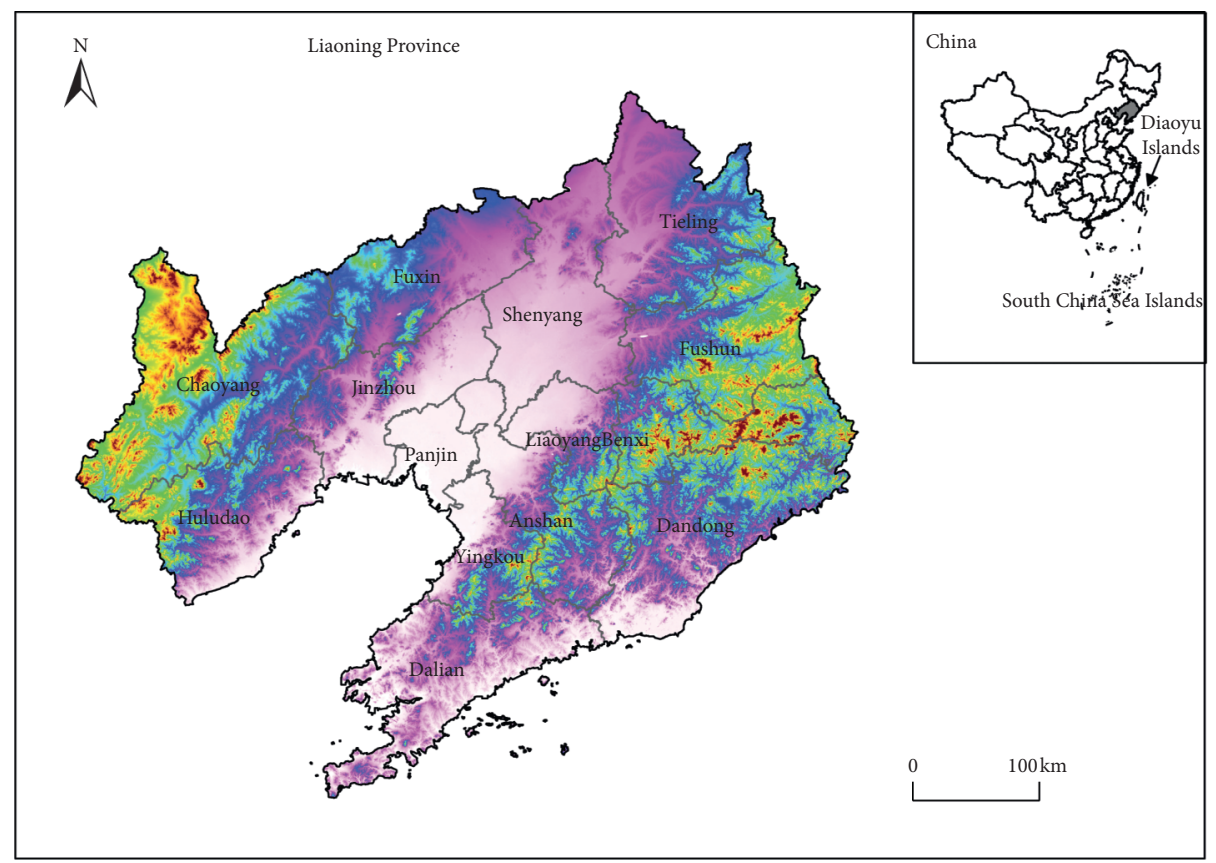

DEM

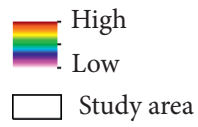

Figure 1: The location of the study area.

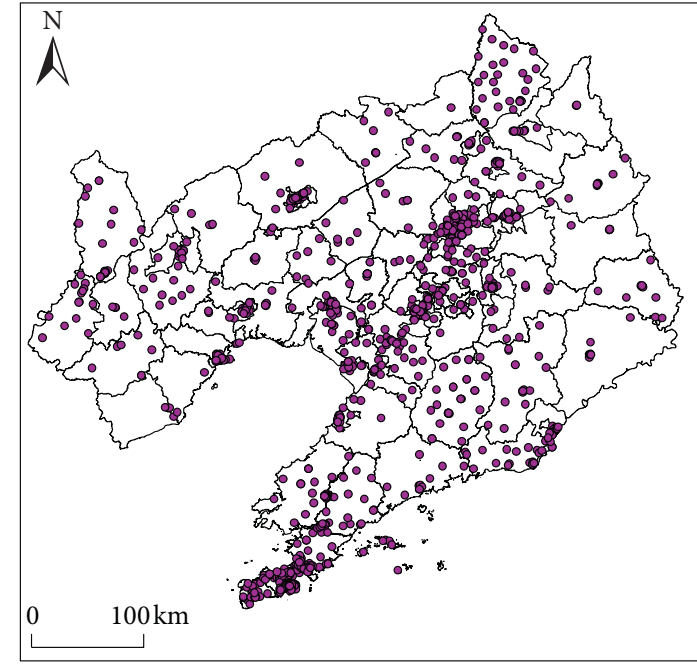

- Primary schools

Study area

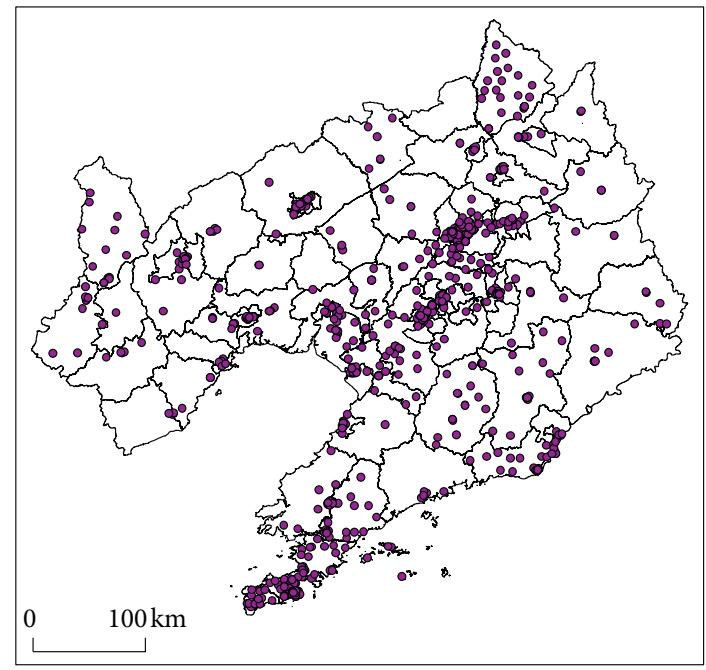

- Secondary schools

Study area

FIGURE 2: Sample points of primary and secondary schools for relocated children of migrant workers.

TABle 1: Data sources and description.

Variable

School data

School coordinates

Remote sensing images

Description

School names, number of children, etc.

School coordinates

DEM of $30 \mathrm{~m}$ resolution
Data source

Government Information Statistics Google Earth

Geospatial Data Cloud 
built-up area, and the number of students in the $l$ th school is $j_{w_{l}}$. Thus, the concentration ratio $\left(\mathrm{CR}_{c}\right)(\%)$ can be expressed as follows:

$$
\mathrm{CR}_{c}=\frac{\sum_{i=1}^{e} j_{c_{i}}}{\sum_{l=1}^{f} j_{w_{l}}+\sum_{i=1}^{e} j_{c_{i}}} \times 100
$$

2.3.2. Kernel Density Estimation. Because the collection of the geographic coordinates of the schools resulted in a large quantity of point data, a kernel density algorithm was used to accurately demonstrate the spatial distribution and agglomeration of the schools as follows. Let $x_{1}, x_{2}, \ldots$, and $x_{n}$ be a sample drawn from a population with a distribution density function $f$, where $f(x)$ symbolizes the estimated value of $f$ at a given point $x$, which can be calculated using the Parzen-Rosenblatt window method.

$$
f(x)=\frac{1}{n h} \sum_{i=1}^{n} k\left(\frac{x-x_{i}}{h}\right),
$$

where $k$ is the kernel function, $h$ indicates the bandwidth and $h>0, n$ represents the number of samples, and $\left(x-x_{i}\right)$ represents the distance from the estimated point $x$ to sample $x_{i}$.

2.3.3. Coefficient of Variation. The coefficient of variation $\left(C_{v}\right)$, a statistical measure of dispersion of observations in the dataset, is the ratio of the standard deviation to the mean. When the dimensions and means of multiple sets of data are different, the degree of dispersion of a dataset can be expressed by the coefficient of variation. A larger coefficient of variation indicates more diversity among the data within the set, suggesting that there are more differences in the number of RCMW among schools, while a smaller coefficient of variation indicates a smaller difference among the data within the set. The calculation formula is as follows:

$$
C_{v}=\frac{\sqrt{(1 / n) \sum_{i=1}^{n}\left(x_{i}-(1 / n) \sum_{i=1}^{n} x_{i}\right)^{2}}}{(1 / n) \sum_{i=1}^{n} x_{i}},
$$

where, $x_{i}$ is the attribute value of element $i$ of the dataset and $n$ is the number of samples in the dataset. The coefficient of variation of the number of RCMW in primary and secondary schools from 2008 to 2017 was used to analyze the evolution of school size. The coefficient of variation of the Voronoi diagram was used to illustrate the spatial evolution of the distribution of schools.

\section{Results}

3.1. Spatial-Temporal Evolution of School Size. In 2008, there were 50,420 RCMW in junior high schools and 149,901 in primary schools; by 2017, the figures had increased to 72,485 (an increase of $43.76 \%$ ) and 173,363 (an increase of 15.65\%), respectively. The maximum number of RCMW in primary schools increased from 1,124 in 2008 to 1,465 in 2017 and the minimum number decreased from 2 to 1 over the same time period. In addition, the number of primary schools with RCMW decreased from 1,137 in 2008 to 1,130 in 2017.

In 2008, there were 431 junior high schools with RCMW (236 schools in the main urban area, 81 schools in high-tech and development zones, and 114 schools in counties). Schools in the main urban area had 125 RCMW on average, while schools in county areas had 81 RCMW on average. From 2008 to 2017, the number of junior high schools with RCMW in each region changed significantly; however, the total number of RCMW showed no noticeable increase. The spatial characteristics of the schools with RCMW are shown in Figure 3.

Using natural breaks classification (Jenks) in ArcGIS, the schools were classified into four levels (I, II, III, and IV) based on the number of RCMW attending. The spatial changes of primary and secondary schools by level in 2008 and 2017 are presented in Figures 3 and 4. In 2008, there were only 11 primary schools in level IV; in 2017, the number increased to 23, including two "mega" schools with more than 1,200 RCMW. From 2008 to 2017, schools in levels I, II, and III increased by 12,15 , and 35 , respectively (Table 2). The number of secondary schools in level IV decreased from 18 in 2008 to 16 in 2017, whereas the schools in levels I, II, and III increased by $52.4 \%, 22.8 \%$, and $42.2 \%$ from 2008 to 2017, respectively (Table 3).

Voronoi diagrams of primary and secondary schools in 2008 and 2017 were created using ArcGIS 10.2 to identify the service coverage of each school. Next, the coefficients of variation of primary and secondary schools in 2008 and 2017 were calculated (Figures 5 and 6). The coefficient of variation characterized the distribution pattern of the nodes; specifically, $C_{v}>64 \%$ indicates a clustered distribution, $C_{v}<33 \%$ indicates a balanced distribution, and $64 \%>C_{V}>33 \%$ indicates a random distribution [44]. According to the principles of Voronoi diagrams, the coefficients of variation of the primary and secondary schools in 2008 were $65.6 \%$ and $66.1 \%$, respectively, while those of the primary and secondary schools in 2017 were $78.4 \%$ and $77.5 \%$, respectively. The increase of the coefficients of variation suggested that the schools were more spatially agglomerated. The overall service coverage per school was reduced, indicating that the number of schools with RCMW increased.

3.1.1. Changes in School Concentration. The concentration ratios of the number of RCMW and their schools in main urban areas, counties, and high-tech and development zones in 2008, 2012, 2014, and 2017 were calculated. The results indicate that the number of RCMW began to concentrate in main urban areas and high-tech and development zones, while the schools were mostly concentrated in main urban areas and counties (Figure 7). In 2008, the four districts with the highest concentration ratios of primary school RCMW in main urban areas were Ganjingzi District (16\%), Dongling District (6.1\%), Tiexi District (Shenyang) (3.4\%), and Xinglongtai District (3.3\%). In 2017, the overall concentration ratio increased, and the four districts with the highest concentration ratios of primary school RCMW in main urban areas were Ganjingzi District (15.5\%), Tiexi District (Shenyang) (15\%), Lianshan District (5\%), and Dadong 


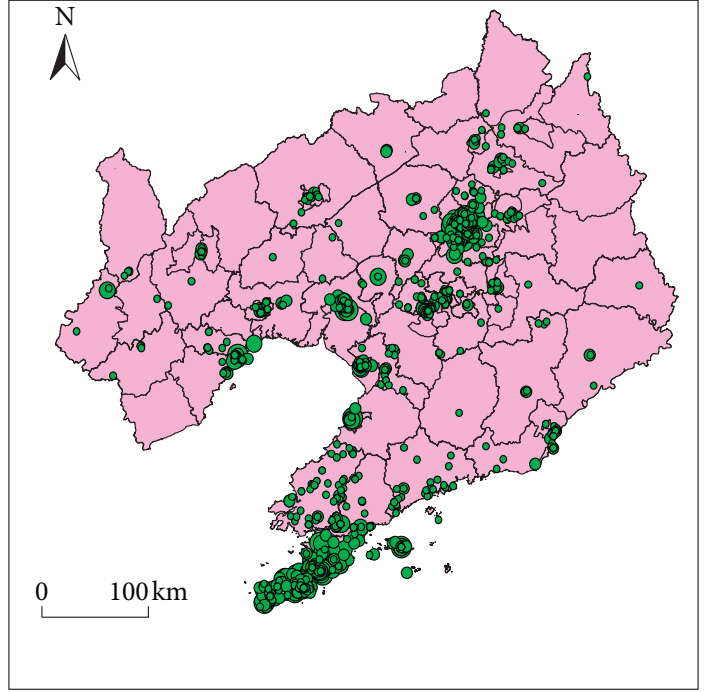

Level

- 1-107

- 108-299
○ $300-584$

585-1224

(a)

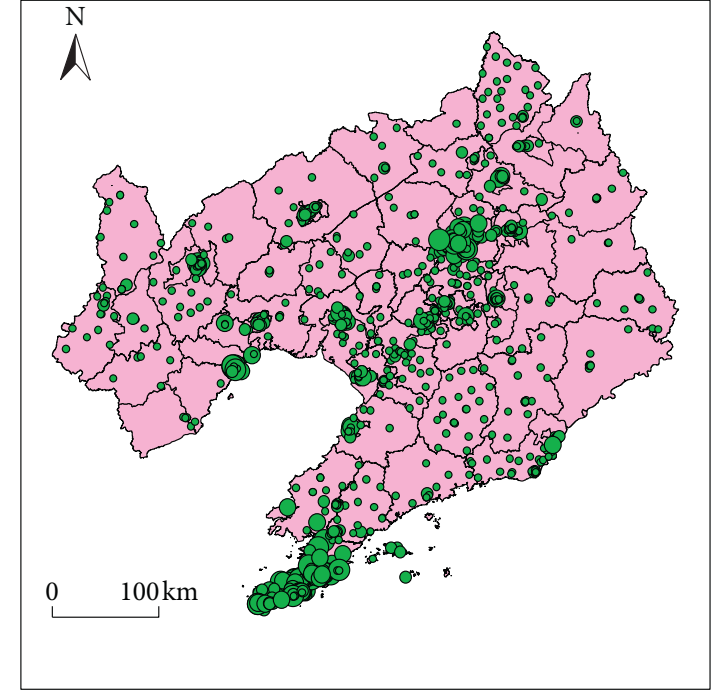

Level

- 1-100

- 101-293
294-610

611-1465

(b)

Figure 3: Spatial variation of the scale of primary schools in Liaoning Province (a) in 2008 and (b) 2017.

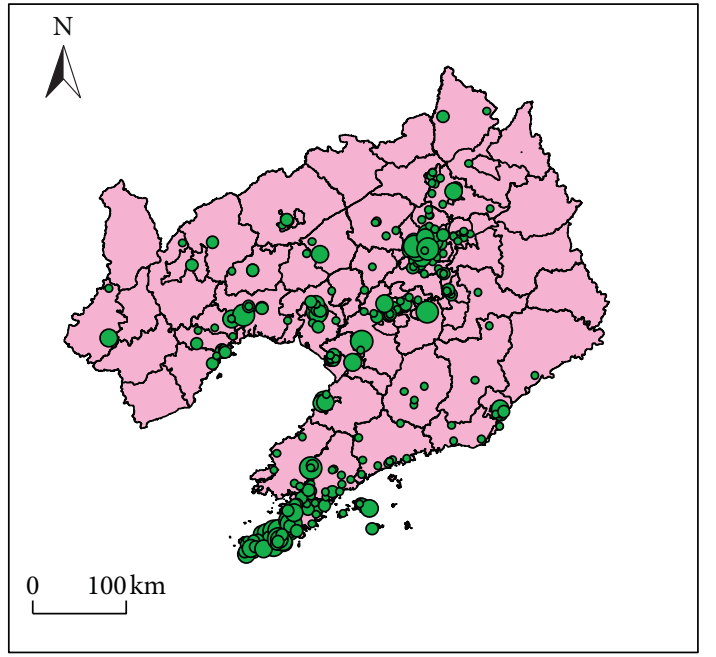

Level

- 1-87

- $88-232$
○ $233-457$

458-886

(a)

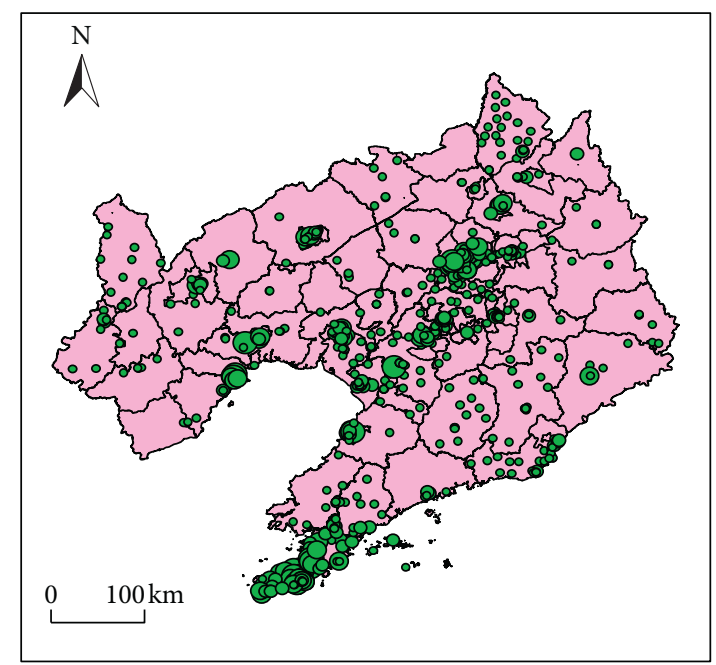

Level

- 1-82

- 83-261

262-561

562-1110

(b)

Figure 4: Spatial variation of the scale of secondary schools in Liaoning Province (a) in 2008 and (b) in 2017.

Table 2: Scale grade of primary schools in Liaoning Province in 2008 and 2017.

\begin{tabular}{ccccc}
\hline & I & II & III & IV \\
\hline 2008 & 696 & 302 & 130 & 11 \\
2017 & 825 & 317 & 165 & 23 \\
\hline
\end{tabular}

Table 3: Scale grade of secondary schools in Liaoning Province in 2008 and 2017.

\begin{tabular}{ccccc}
\hline & I & II & III & IV \\
\hline 2008 & 254 & 118 & 41 & 18 \\
2017 & 534 & 153 & 71 & 16 \\
\hline
\end{tabular}




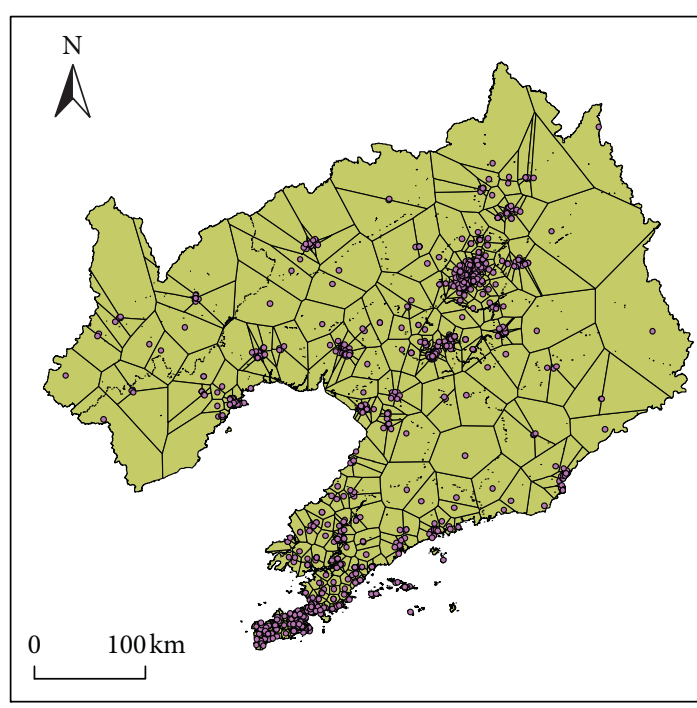

(a)

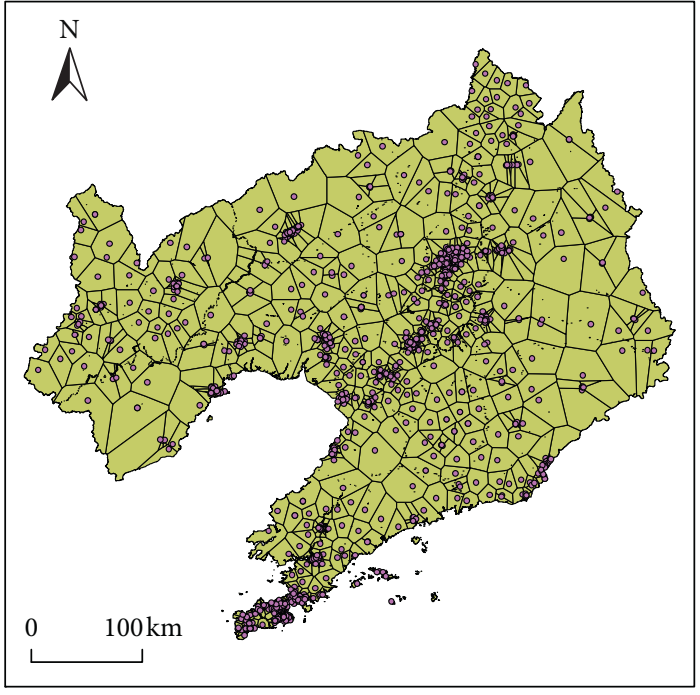

(b)

Figure 5: Voronoi maps of primary schools in Liaoning Province (a) in 2008 and (b) in 2017.

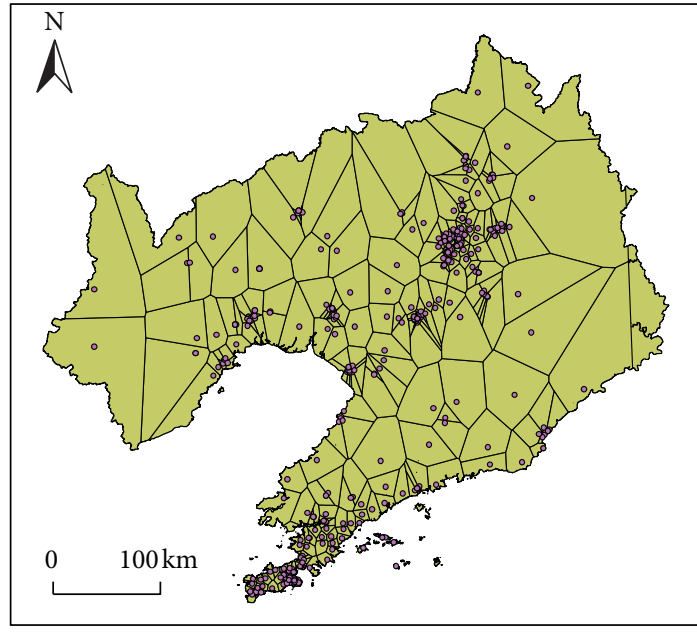

(a)

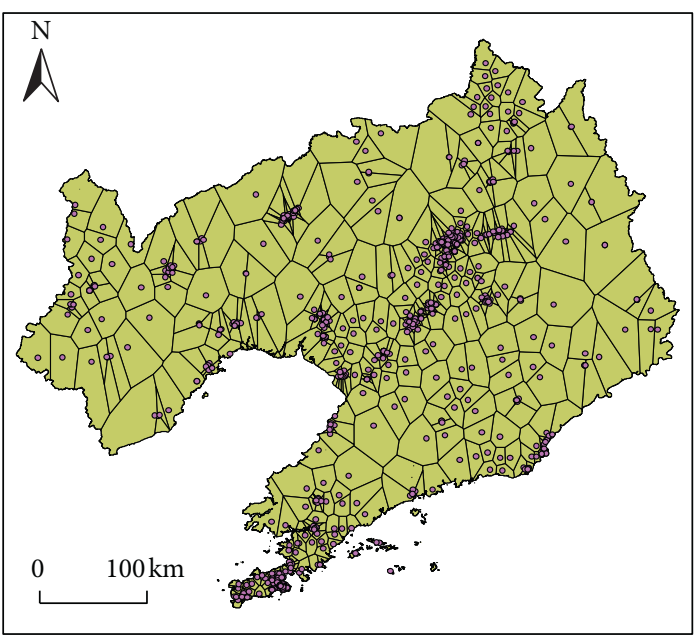

(b)

Figure 6: Voronoi maps of secondary schools in Liaoning Province (a) in 2008 and (b) in 2017.

District (4.1\%). The districts with a high concentration of RCMW were mainly located in cities that had experienced rapid urbanization, such as Shenyang and Dalian. Compared to 2008 , the concentration ratios of primary schools in main urban areas and counties in 2017 increased by $1.6 \%$ and $1.7 \%$, respectively; however, the concentration ratio in hightech and development zones decreased by $2.1 \%$. In 2017, the concentration ratio of secondary schools in main urban areas and high-tech and development zones decreased by $9.5 \%$ and $3.5 \%$, respectively, compared to 2008 , while the concentration ratio in counties increased by $12.8 \%$ (Table 4 ).

3.1.2. Kernel Density and Spatial Agglomeration. The KDE method and ArcGIS 10.2 were employed to achieve data visualization of the spatial agglomeration of RCMW and their primary and secondary schools. Figure 8 shows that the spatial-temporal evolution of the number of RCMW and their primary and secondary schools tended to converge. The maximum density values of the number of children and schools showed various levels of increase over the research period and were mainly distributed in the central urban areas of prefecture-level cities. These findings indicated that the distribution of schools with RCMW had a tendency to cluster toward the central urban areas. In addition, the kernel density of the number of children and schools varied greatly among counties. Their kernel density in the majority of the counties and county-level cities decreased over the research period, although it increased in a limited number of county-level cities with relatively rapid urbanization, such as Kuandian Manchu Autonomous County, Huanren Manchu Autonomous County, and Xifeng County. 


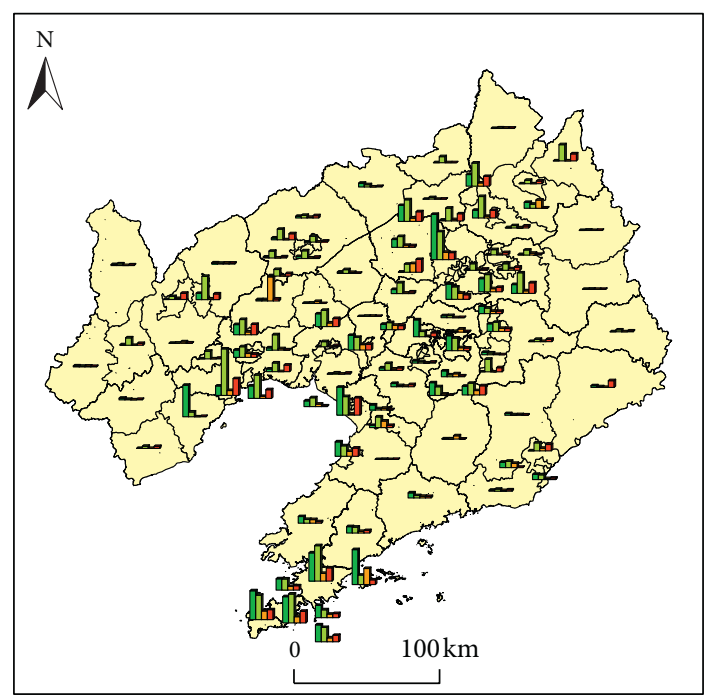

RCMW in primary schools in 2008

RCMW in primary schools in 2017

RCMW in secondary schools in 2008

RCMW in secondary schools in 2017

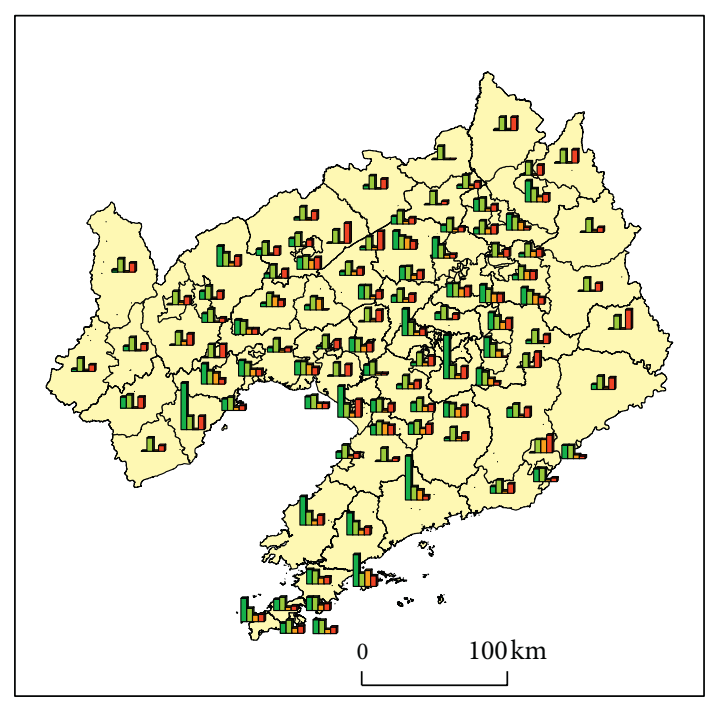

RCMW's primary schools in 2008

RCMW's primary schools in 2017

RCMW's secondary schools in 2008

RCMW's secondary schools in 2017

FIgURE 7: Concentration ratio of primary and secondary schools' RCMW and their schools in Liaoning Province in 2008 and 2017.

TABLE 4: Concentration ratio of primary and secondary schools' RCMW and schools with RCMW in main urban areas, counties, and hightech zones in Liaoning Province in 2008 and 2017.

\begin{tabular}{lcccc}
\hline & Primary schools (2008) & Primary schools (2017) & Secondary schools (2008) & Secondary schools (2017) \\
\hline RCMW (urban areas) & 55.9 & 63.5 & 53.1 & 63.2 \\
RCMW (counties) & 11 & 6 & 17.7 & 8.9 \\
RCMW (high-tech zones) & 33.1 & 30.5 & 29.2 & 27.9 \\
Schools (urban areas) & 48.9 & 50.5 & 56.3 & 46.8 \\
Schools (counties) & 32.7 & 34.4 & 25.4 & 38.2 \\
Schools (high-tech zones) & 18.4 & 16.1 & 18.3 & 15 \\
\hline
\end{tabular}

Further, the spatial distribution of the number of children and schools evolved from a gradually stretched pattern to clustered, discretized, and polar nucleus-shaped patterns. The polar nucleus-shaped patterns were most prominent in the main urban areas and fringe areas of Shenyang and Dalian. Other regions also showed a relatively less prominent polar nucleus. The clusters were mainly distributed in the central urban areas of the prefecture-level cities. Kernel density values underwent the following changes: areas with low density values shrank, areas with medium density values expanded, and areas with high density values tended to cluster. In addition, areas with high density values increased. In 2008, Liaoning was less urbanized, the development of schools in cities and counties was relatively balanced, and the distribution of RCMW in primary and secondary schools was relatively even. However, in 2017, with the acceleration of urbanization, the spatial distribution of the number of RCMW in schools became imbalanced, and the majority of children tended to cluster in certain regions. As a result, kernel density manifested clustered and polar nucleus-shaped patterns. In addition, the distributions in counties, cities, and provincial capitals were noticeably different, forming a 3-level gradient pattern.

3.1.3. Unbalanced Development of Primary and Secondary Schools between Urban and Rural Areas. Balanced development of primary and secondary education means that every school-age child has equal opportunity to receive education of equal quality in similar environments, and the allocation of educational resources is reasonable among regions, urban and rural areas, and schools. With the continuous acceleration of urbanization, high-quality educational resources, job opportunities, and welfare benefits in urban areas have influenced a large number of rural households to migrate to cities. As a result, more children were transferred from schools in rural areas to schools in urban areas, leading to a reduction in the number of students in county schools and an increased gap in student numbers between urban and rural schools. Some schools in urban areas have an exceptionally larger number of students compared to other schools. Based on "Chengshi Putong Zhong Xiao Xuexiao Jianshe Biaozhun (the Standards for the Construction of General Primary and Secondary Schools in 


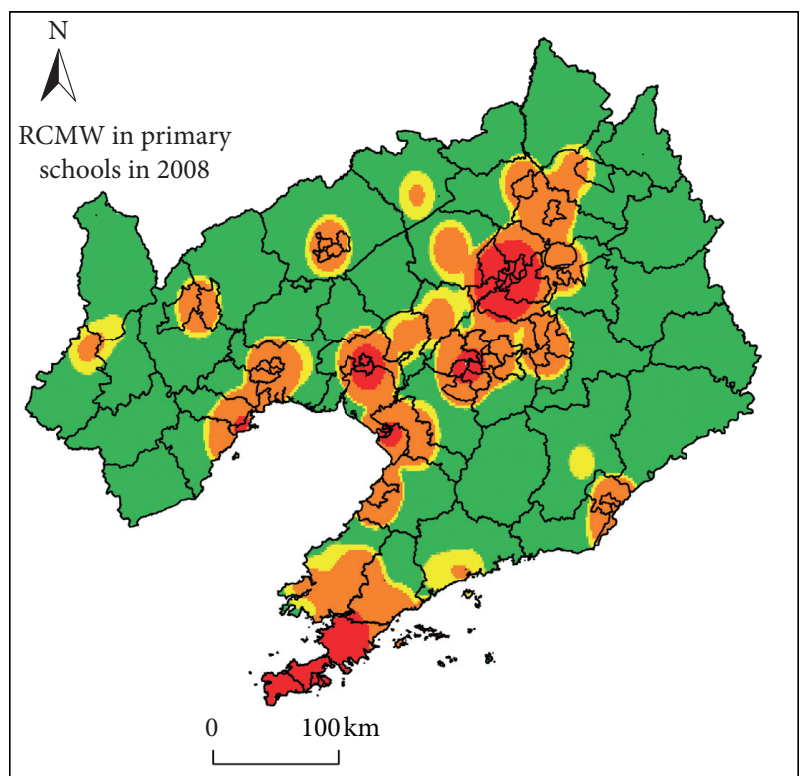

0-1572

1573-1673

1674-3245

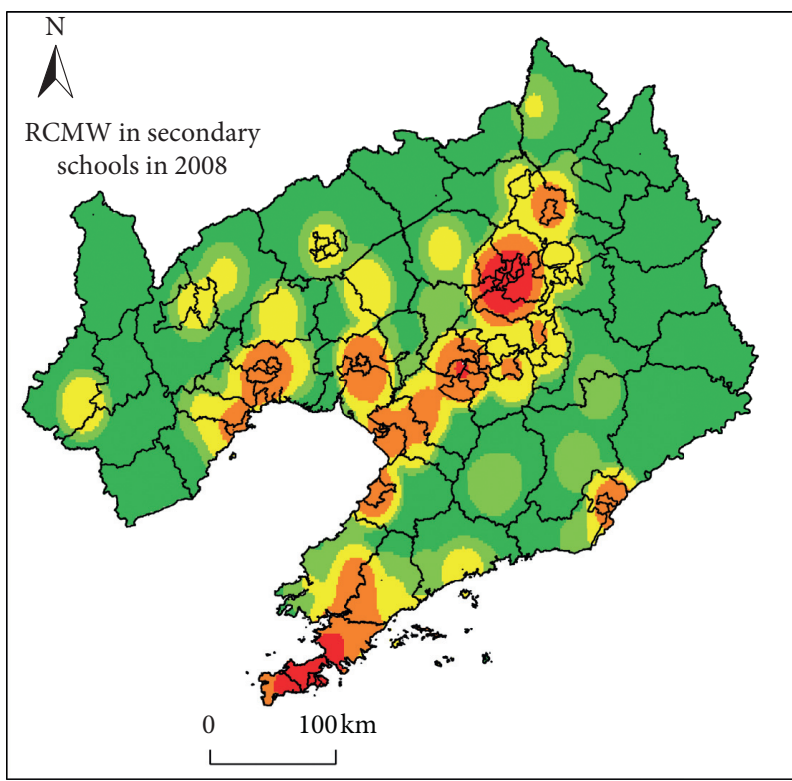

- 0-161

162-942

943-4711
$3246-27674$

27675-407254

4712-22897

22898-110666

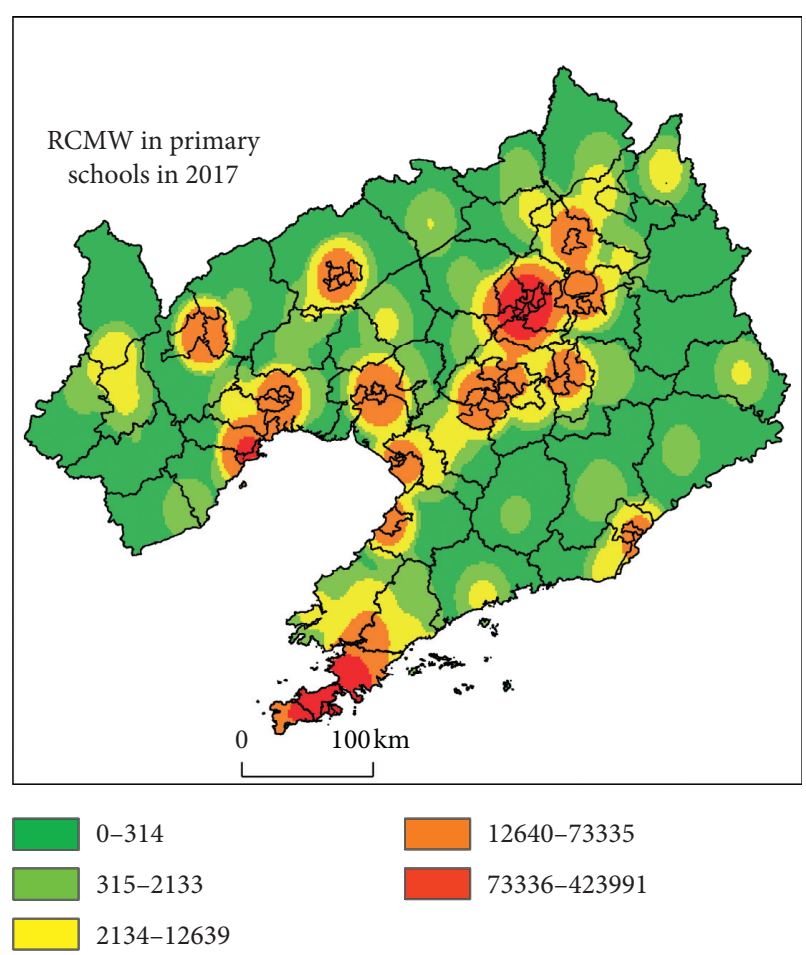

(a)

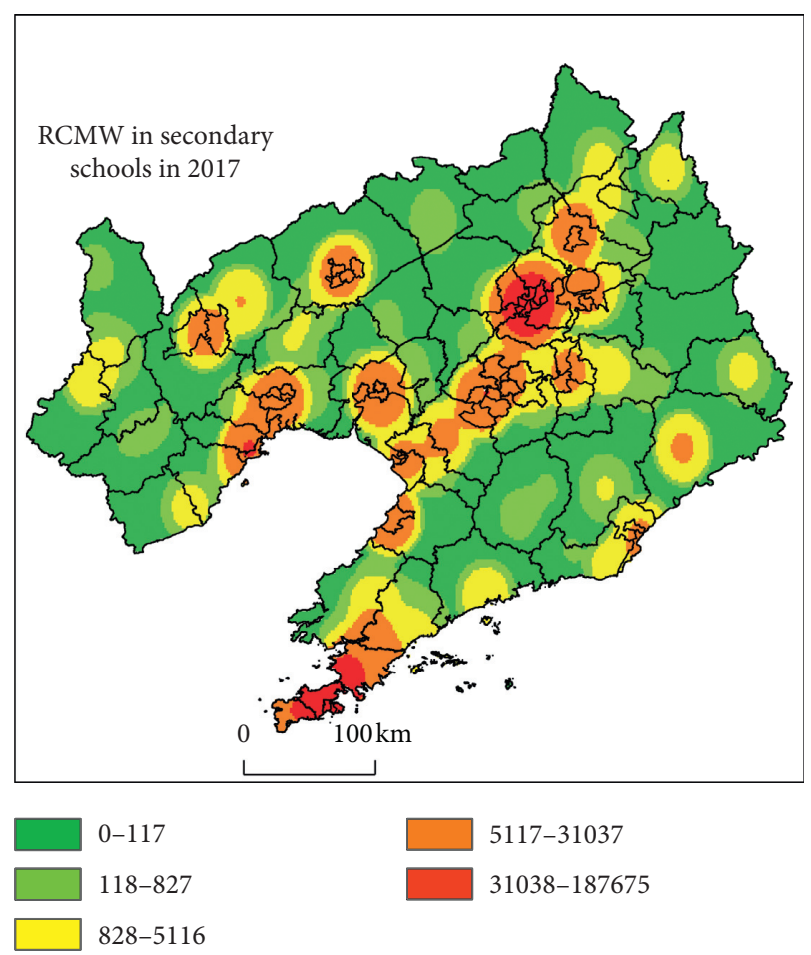

(b)

Figure 8: Continued. 

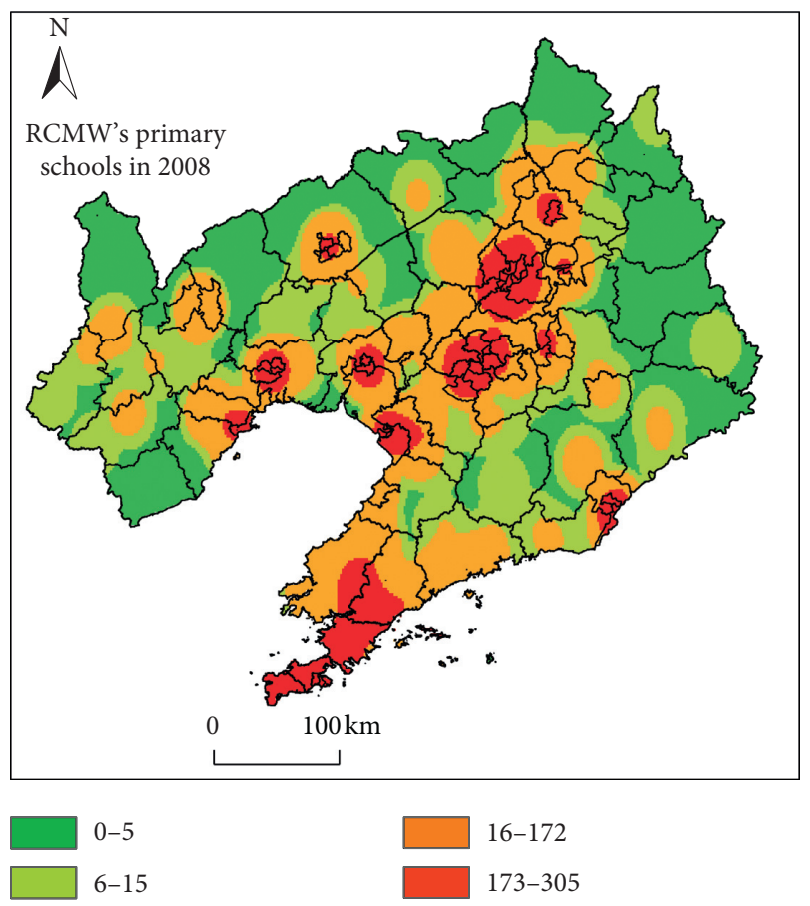
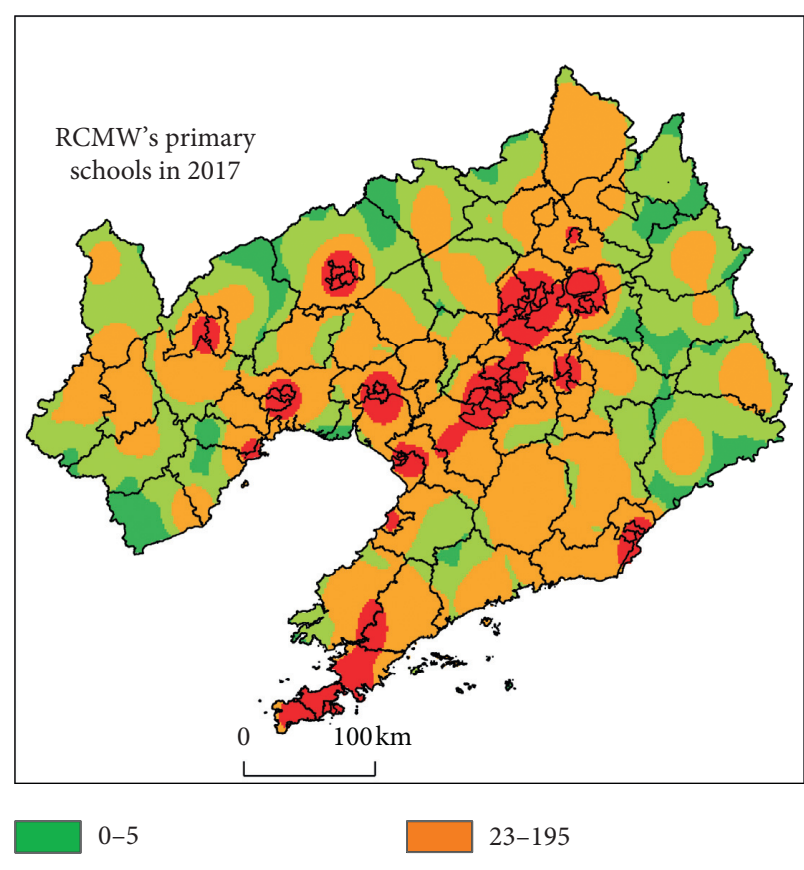

6-22

23-195

196-336

(c)
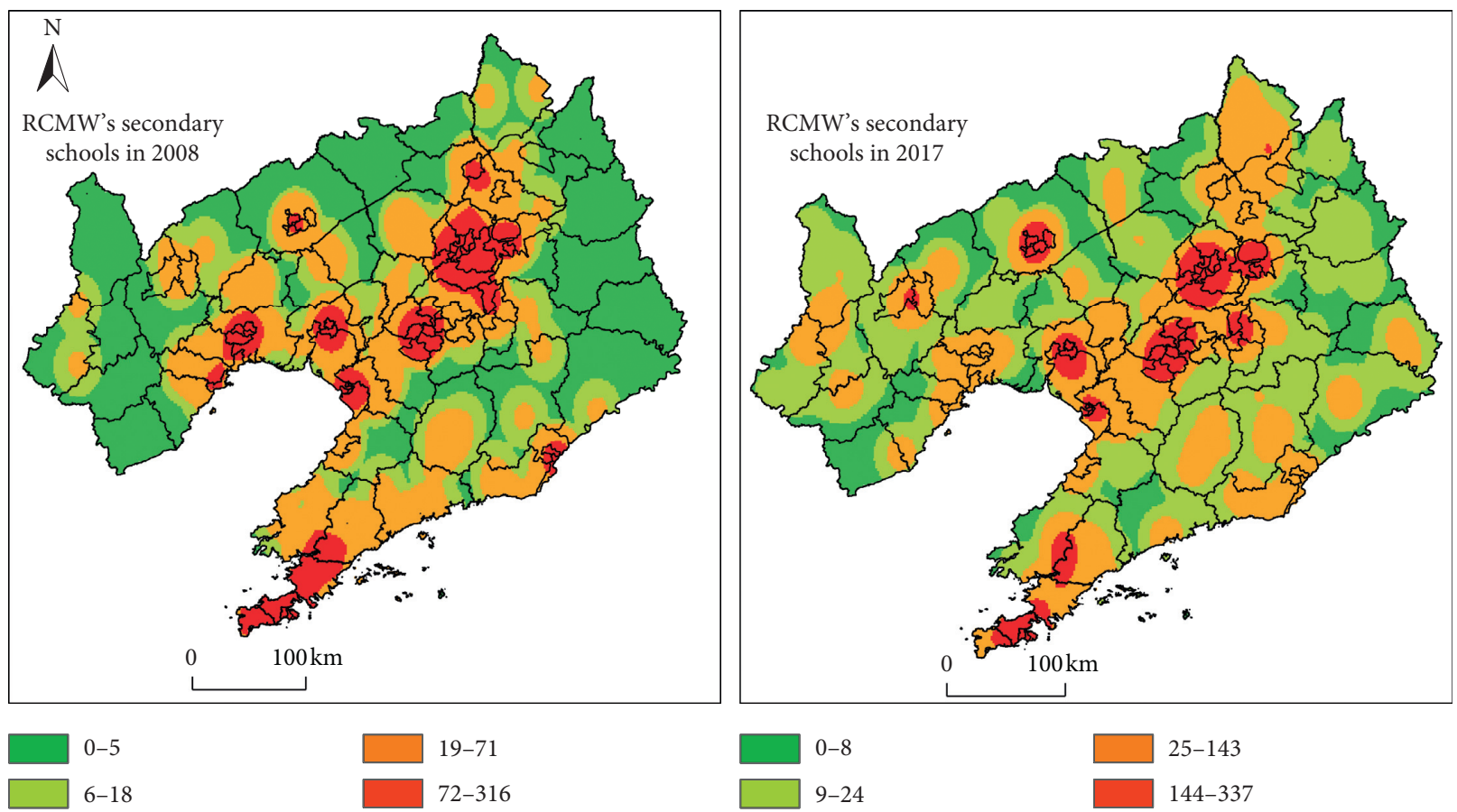

(d)

Figure 8: Distribution density map of RCMW and their primary and secondary schools in Liaoning Province in 2008 and 2017.

Urban Areas)," "Nongcun Putong Zhong Xiao Xuexiao Jianshe Biaozhun (the Standards for the Construction of General Primary and Secondary Schools in Rural Areas)," and the actual situation in Liaoning, the primary and secondary schools were categorized based on the number of RCMW. Specifically, primary schools with fewer than 200, 200 to 500, 501 to 700, and more than 700 RCMW were defined as "small," "medium- sized," "large," and "super-large" schools, respectively; secondary schools with fewer than 150, 150 to 300, 301 to 500, and more than 500 RCMW were defined as "small," "mediumsized," "large," and "super-large" schools, respectively.

In 2008, there were 29 super-large and 30 large schools, whereas in 2017, there were 58 super-large and 90 large schools. In other words, the number of super-large and large 
TABLE 5: The coefficients of variation of RCMW in primary and secondary schools in Liaoning Province.

\begin{tabular}{lcccc}
\hline & 2008 & 2011 & 2014 & 2017 \\
\hline Primary schools & 132 & 135 & 145 & 147 \\
Secondary schools & 122 & 132 & 161 & 163 \\
\hline
\end{tabular}

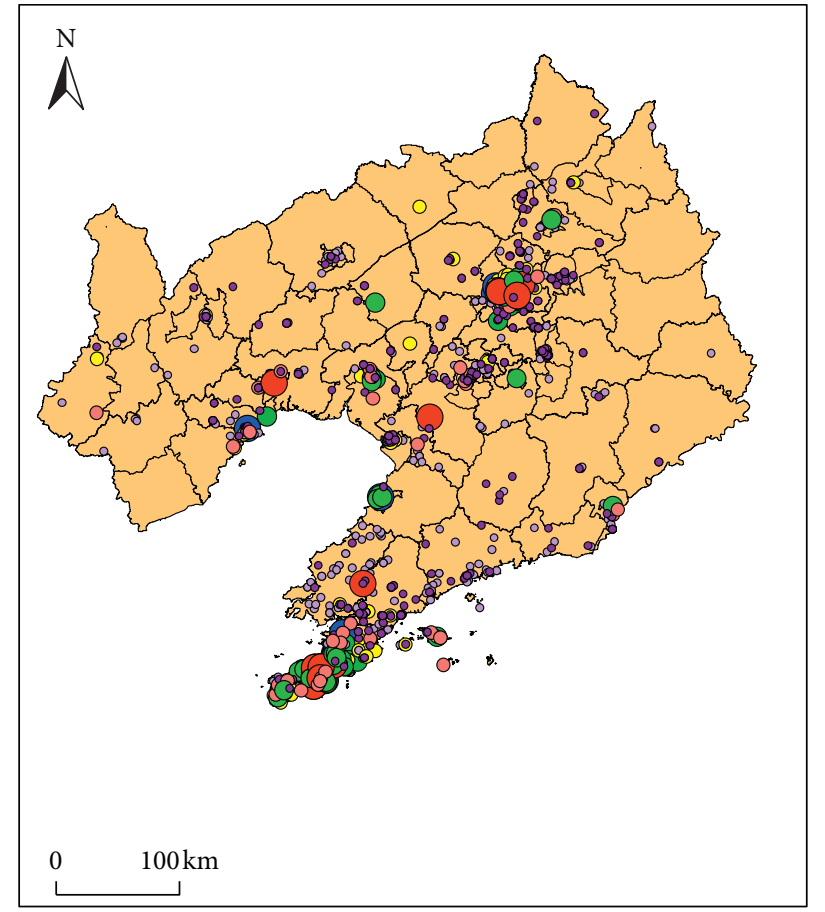

- Small secondary schools

- Medium-sized secondary schools

Large secondary schools

Super-large secondary schools
- Small primary schools

- Medium-sized primary schools

Large primary schools

Super-large primary schools

(a)

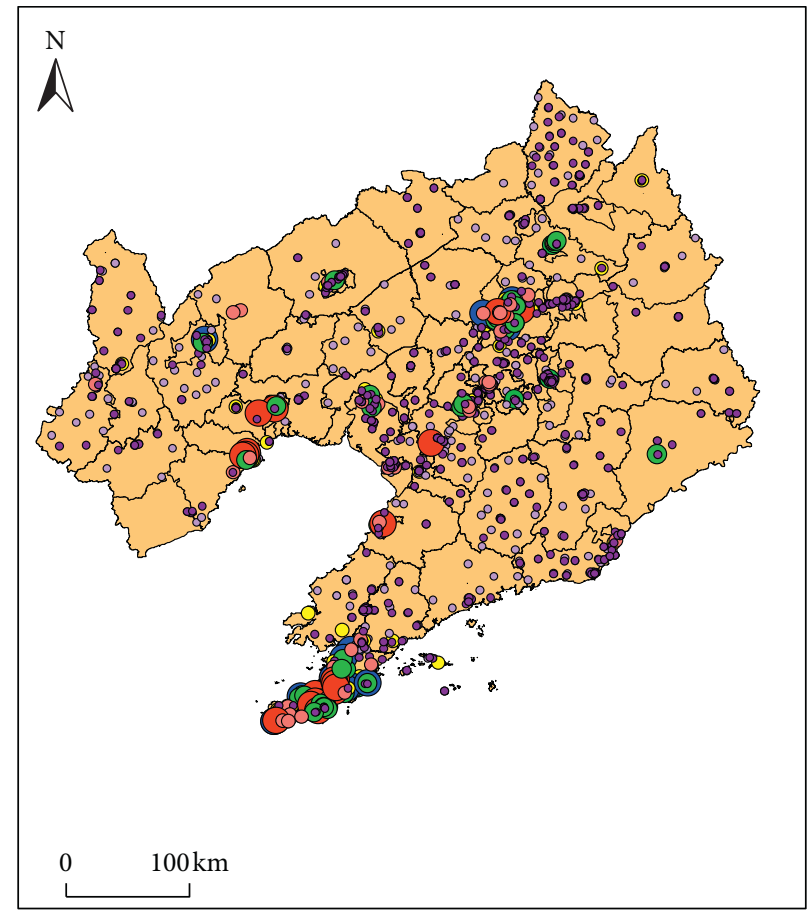
- Small secondary schools
- Medium-sized secondary schools
Large secondary schools
Super-large secondary schools

- Small primary schools

- Medium-sized primary schools

$\bigcirc$ Large primary schools

Super-large primary schools

(b)

Figure 9: The spatial distribution of "small school" and "large school" in Liaoning Province in (a) 2008 and (b) 2017.

schools doubled and tripled in size, respectively, between 2008 and 2017. In 2008, there were 221 medium-sized and 1,231 small schools, while in 2017 , there were 460 mediumsized and 1,853 small schools.

The coefficients of variation of RCMW in primary and secondary schools in the province are shown in Table 5. The coefficient of variation gradually increased from 2008 to 2017 , indicating that the overall scale of RCMW children in primary and secondary schools gradually increased. However, the growth of the number of primary school students was smaller than that of the number of secondary school students. In addition, the changes in the number of children presented as an inverted U-shape. The rate of growth in the number of RCMW between 2008 and 2011 and between 2014 and 2017 was notably slower than that between 2011 and 2014. From 2008 to 2011, the differences in the number of RCMW among primary schools were greater than those among junior high schools. However, from 2014 to 2017, the difference in the number of RCMW in junior high schools gradually increased and exceeded the differences among the primary schools. In addition, the majority of RCMW tended to cluster in cities, leading to an increase in the number of large and super-large schools in urban areas and a reduction in the per capita educational resources for local students in these areas. The majority of schools in counties were small and medium-sized, leading to a further reduction of educational resources in counties and more pronounced differences in the development of educational resources between urban and rural areas (Figure 9).

\subsection{Mechanisms behind the Spatial Differences}

3.2.1. Effect of Regional Economic Development on Schools. Differences in economic development are the major factor of the imbalanced spatial distribution of primary and secondary schools within the province. Due to factors such as 
geographic location and industry structure, the economic development of counties in Liaoning varied significantly, leading to an imbalance in the investment of education and construction funding between schools; key schools were highly concentrated in regions with rapid economic development. Moreover, economic development tended to attract a large number of migrant workers and high-quality teachers. The transfer of the children of these workers into the key primary and secondary schools facilitated the formation of large and super-large schools. Higher-quality teachers were generally attracted to key schools, which widened the gap in teaching quality among schools. Thus, the differences in regional economic development accelerated unbalanced growth in the number of RCMW and in the development of educational resources among counties.

\subsubsection{Demand for Educational Resources by Population Size.} Population size was the core element affecting the spatial distribution of primary and secondary schools, as large populations provide a stable source of students. Therefore, the concentration of primary and secondary schools in a given region was positively correlated to its population size. A large school-age population required more primary and secondary schools, which promoted the rapid development of education quality, leading to the uncoordinated development of primary and secondary education among counties with different population sizes. Moreover, the increase in population size accelerated the process of urbanization, attracting more RCMW into the area, which made the imbalanced spatial distribution of primary and secondary educational resources more prominent.

3.2.3. Guidance of Government Policies. The implementation of corresponding policies, such as the "The National Outline for Medium and Long-term Education Reform and Development (2010-2020)" and "Decisions of the State Council on Further Strengthening the Work on Rural Education," had a certain impact on the spatial distribution of primary and secondary schools for RCMW and were of great significance to promote the balanced development of primary and secondary education. In addition, the previous family planning policy and newly implemented two-children policy had a strong impact on the population structure and the number of students in the region, which indirectly affected the spatial distribution of RCMW in primary and secondary schools.

\section{Conclusion}

Using data related to the RCMW in schools in Liaoning from 2008 to 2017, concentration ratio, kernel density, and coefficient of variation methods, this study explored the spatial-temporal characteristics and balance of the distribution of primary and secondary school resources. The findings are as follows:
(1) From 2008 to 2017, the total number of RCMW enrolled in primary and secondary schools in Liaoning presented an overall growing trend; however, the growth of the number of primary school children $(15.65 \%)$ was smaller than that of junior high school children (43.76\%). The number of primary schools with these children remained stable, while the number of junior high schools decreased by $44.3 \%$. The concentration ratio of these children in urban primary and secondary schools increased from $55.9 \%$ to $63.5 \%$, while the concentration ratio in counties and high-tech and development zones decreased from $11 \%$ and $33.1 \%$ to $6 \%$ and $30.5 \%$, respectively.

(2) The spatial distribution pattern of the children and their schools evolved from a balanced stretched pattern toward polar nucleus-shaped, clustered, and discretized patterns. Specifically, larger schools tended to cluster, while the distribution of small schools tended to scatter. The distribution in main urban areas and urban fringe areas of Shenyang and Dalian presented prominent polar nucleus shapes, forming a dual-core structure within the province. In addition, the distribution among counties, cities, and provincial capitals formed a 3-level gradient pattern. Areas with low density values became smaller, areas with medium density values expanded, and areas with high density values tended to cluster and increased.

(3) Large and super-large schools emerged in urban areas, while schools in counties were usually small to medium-sized. From 2008 to 2017, the coefficients of variation of relocated children in primary and secondary schools increased from 132 and 122 to 147 and 163 , respectively.

(4) The major factors that affected the distribution patterns of schools for these children included: (1) regional economic development, (2) population size, and (3) government policies. The combined effects of the three mechanisms further exacerbated the imbalance in the spatial distribution of educational resources for these children.

\section{Discussion}

This study employed concentration ratio, kernel density analysis, and the coefficient of variation to investigate the spatial-temporal evolution and balance of primary and secondary schools for RCMW in Liaoning. The findings were of great significance for the rational distribution and balanced development of primary and secondary schools in the province. In contrast to existing research from home and abroad, the present study provides the following contributions: (1) New research perspectives: the majority of past studies tended to analyze the overall spatial characteristics of primary and secondary schools from a macroperspective. Research on the spatial-temporal evolution and balance of schools from the perspective of RCMW under the 
background of new-type urbanization was limited [45-47]. (2) New research data: existing research mostly used questionnaire surveys, statistical yearbooks, and field observations to collect data. However, the data used in this study were extracted from government data pools, which improved the accuracy of the research data $[48,49]$. (3) New research areas: existing studies mainly investigated the topic at community, county, or city levels with limited studies at the provincial level [50-52].

Nevertheless, this study has the following limitations: (1) The closing of old schools, the opening of new schools, and the relocation of existing schools might affect their spatial distribution. (2) Changes in the division of administrative zones and establishment of high-tech and development zones (such as converting Pulandian from a city to district and establishing Jinpu New District and the Puwan Economic Zone) might affect the spatial differences of primary and secondary schools. However, these changes were not controlled for in this study. Future studies may account for these limitations.

\section{Data Availability}

The data used to support the findings of this study are available from the corresponding author upon request.

\section{Conflicts of Interest}

The authors declare that there are no conflicts of interest regarding the publication of this paper.

\section{Acknowledgments}

This study was funded by 2018 National Social Science Fund of China Project: Research on Resources Allocation of Compulsory Education Based on School-age Population Flow Trend Prediction (Grant no. 18BRK037), as well as General Topic of the "13th Five-year" Education Science Planning of Liaoning Province in 2018: Research on the Allocation of Compulsory Education Resources Based on the Prediction of School-age Population Mobility Trend in Liaoning Province (Grant no. JG18CB390).

\section{References}

[1] M. Colas and S. Ge, "Transformations in China's internal labor migration and hukou system," Journal of Labor Research, vol. 40, no. 3, pp. 296-331, 2019.

[2] X. Wang and N. Weaver, "Surplus labour and urbanization in China," Eurasian Economic Review, vol. 3, pp. 84-97, 2013.

[3] Z. Lijun, Study on the Methods of Land Use Master Planning Map, East China Normal University, Shanghai, China, 2011.

[4] S. Müller, S. Tscharaktschiew, and K. Haase, "Travel-to-school mode choice modelling and patterns of school choice in urban areas," Journal of Transport Geography, vol. 16, no. 5, pp. 342-357, 2008.

[5] A. K. Yarlagadda and S. Srinivasan, "Modeling children's school travel mode and parental escort decisions," Transportation, vol. 35, no. 2, pp. 201-218, 2008.

[6] H.-P. Hsu and J.-D. Saphores, "Impacts of parental gender and attitudes on children's school travel mode and parental chauffeuring behavior: results for California based on the 2009 national household travel survey," Transportation, vol. 41, no. 3, pp. 543-565, 2014.

[7] M. Hidalgo-Hidalgo and I. Iturbe-Ormaetxe, "Should we transfer resources from college to basic education?" Journal of Economics, vol. 105, no. 1, pp. 1-27, 2012.

[8] A. Balestrino, L. Grazzini, and A. Luporini, "A normative justification of compulsory education," Journal of Population Economics, vol. 30, no. 2, pp. 537-567, 2017.

[9] C. Doniskeller, B. O. Hara Miklavic, and J. C. Fairman, "Improving educational opportunity and equity through school district consolidation in maine," Maine Policy Review, vol. 22, no. 2, pp. 42-54, 2013.

[10] J. Malczewski and M. Jackson, "Multicriteria spatial allocation of educational resources: an overview," Socio-Economic Planning Sciences, vol. 34, no. 3, pp. 219-235, 2000.

[11] R. K. Norton, "Planning for school facilities," Journal of Planning Education and Research, vol. 26, no. 4, pp. 478-496, 2007.

[12] J. Pearce, "Techniques for defining school catchment areas for comparison with census data," Computers, Environment and Urban Systems, vol. 24, no. 4, pp. 283-303, 2000.

[13] M. Moussa, Y. Mostafa, and A. A. Elwafa, "School site selection process," Procedia Environmental Sciences, vol. 37, pp. 282-293, 2017.

[14] C. Zhan, "School choice programs and location choices of private schools," Economic Inquiry, vol. 56, no. 3, pp. 1622$1645,2018$.

[15] D. Grimaldi and V. Fernandez, "The road to school. The barcelona case," Cities, vol. 65, no. 5, pp. 24-31, 2017.

[16] P. F. Hanley, "Transportation cost changes with statewide school district consolidation," Socio-Economic Planning Sciences, vol. 41, no. 2, pp. 163-179, 2007.

[17] E. Andersson, B. Malmberg, and J. Östh, "Travel-to-school distances in Sweden 2000-2006: changing school geography with equality implications," Journal of Transport Geography, vol. 23, pp. 35-43, 2012.

[18] K. Boussauw, M. van Meeteren, and F. Witlox, "Short trips and central places: the home-school distances in the Flemish primary education system (Belgium)," Applied Geography, vol. 53, pp. 311-322, 2014.

[19] H. Mitchell, R. A. Kearns, and D. C. A. Collins, "Nuances of neighbourhood: children's perceptions of the space between home and school in Auckland, New Zealand," Geoforum, vol. 38, no. 4, pp. 614-627, 2017.

[20] V. Jenjekwa, "Access and quality in education in resettlement schools:the case study of Zvivingwi secondary school in gutu district, masvingo province in Zimbabwe," International Journal of Educational Administration and Policy Studies, vol. 5, no. 2, pp. 15-21, 2013.

[21] H. Jin and Y. Lu, "The relationship between obesity and socioeconomic status among Texas school children and its spatial variation," Applied Geography, vol. 79, pp. 143-152, 2017.

[22] J. Yang, A. Guo, Y. Li, Y. Zhang, and X. Li, "Simulation of landscape spatial layout evolution in rural-urban fringe areas: a case study of Ganjingzi district," GIScience \& Remote Sensing, vol. 56, no. 3, pp. 388-405, 2019.

[23] J. Yang, Y. Guan, J. Xia, C. Jin, and X. Li, "Spatiotemporal variation characteristics of green space ecosystem service value at urban fringes: a case study on Ganjingzi district in Dalian, China," Science of the Total Environment, vol. 639, pp. 1453-1461, 2018. 
[24] J. Yang, L. Chuang, Y. Li, J. Xi, Q. Ge, and X. Li, "Urban green space, uneven development and accessibility: a case of Dalian's xigang district," Chinese Geographical Science, vol. 25, no. 5, pp. 644-656, 2015.

[25] S. Jin, J. Yang, E. Wang, and J. Liu, "The influence of highspeed rail on ice-snow tourism in northeastern China," Tourism Management, vol. 78, Article ID 104070, 2020.

[26] D. Dai, C. Zhou, and C. Ye, "Spatial-temporal characteristics and factors influencing commuting activities of middle-class residents in Guangzhou city, China," Chinese Geographical Science, vol. 26, no. 3, pp. 410-428, 2016.

[27] B. Bo, W. Lin, and Q. Chen, "Exploring socio-spatial equity in basic educational serve distribution in Beijing municipality," Urban Development Studies, vol. 24, no. 10, pp. 70-78, 2017, in Chinese.

[28] P.-F. Yang, R. He, and H.-B. Hou, "Distribution of primary school based on spatial network comprehensive model in lowincome mountainous cities: a case study in Wanyuan, China," Journal of Mountain Science, vol. 14, no. 10, pp. 2082-2096, 2017.

[29] H. Wen, Y. Xiao, and L. Zhang, "School district, education quality, and housing price: evidence from a natural experiment in Hangzhou, China," Cities, vol. 66, no. 6, pp. 72-80, 2017.

[30] S. Y. He, "Will you escort your child to school? the effect of spatial and temporal constraints of parental employment," Applied Geography, vol. 42, no. 8, pp. 116-123, 2013.

[31] S. Liu and X. Zhao, "How far is educational equality for China? analysing the policy implementation of education for migrant children," Education Resource Policy Practice, vol. 18, no. 1, pp. 59-74, 2019.

[32] X. Wang, X. Chen, and J. Jiao, "Accessibility of urban primary schools based on family travel behavior: a case study of xi' an," City Planning Review, vol. 39, no. 12, pp. 64-72, 2015.

[33] Y. Kong, X. Li, and X. Zhang, "Analysis of spatial accessibility for school redistricting in rural China: a case study of the secondary schools in Gongyi city, Henan province," Journal of Remote Sensing, vol. 12, no. 5, pp. 800-809, 2008, in Chinese.

[34] R. Bifulco, H. F. Ladd, and S. L. Ross, "Public school choice and integration evidence from Durham, North Carolina," Social Science Research, vol. 38, no. 1, pp. 71-85, 2009.

[35] Y. Peng and Z. Wang, "Space operation of rural primary and secondary school location," Acta Geographica Sinica, vol. 68, no. 10, pp. 1411-1417, 2013, in Chinese.

[36] F. Araya, R. Dell, P. Donoso, V. Marianov, F. Martínez, and A. Weintraub, "Optimizing location and size of rural schools in Chile," International Transactions in Operational Research, vol. 19, no. 5, pp. 695-710, 2012.

[37] L. Stiefel, B. Elbel, M. Pflugh Prescott, S. Aneja, and A. E. Schwartz, "School wellness programs: magnitude and distribution in New York city public schools," Journal of School Health, vol. 87, no. 1, pp. 3-11, 2017.

[38] W. D. Duncombe and J. Yinger, "School district consolidation: the benefits and cost," School Administrator, vol. 67, no. 5, pp. 10-17, 2010.

[39] D. Zhang, "The detrimental effects of social capital on the balanced development of compulsory education and their governance," Frontiers of Education in China, vol. 4, no. 3, pp. 390-412, 2009.

[40] M. Tang, Y. Chen, Y. Liu et al., "Equalization of basic education facilities in Ningbo city: a case study of public elementary school," Modern Urban Research, vol. 5, pp. 24-32, 2018, in Chinease.
[41] X. Fan and P. Peng, "Educational equity and institutional safeguards," Frontiers of Education in China, vol. 3, no. 3, pp. 321-330, 2008.

[42] T. Lin, K. Holmes, and M. Zhang, "Better educational inclusion of migrant children in urban school? exploring the influences of the population control policy in large Chinese cities," Asian Social Work and Policy Review, vol. 12, no. 1, pp. 54-62, 2018.

[43] Y. Hu and J. Yinger, "The impact of school district consolidation on housing prices," National Tax Journal, vol. 61, no. 4, Part 1, pp. 609-633, 2008.

[44] Y. Liu and Li Yang, "Analysis of China's FDI regional differences based on Moran'I index," Journal of Chongqing University:Social Science Edition, vol. 17, no. 1, pp. 1-8, 2011, in Chinese.

[45] T. Tu, D. Yan, J. Chen et al., "Evolution of compulsory educational facilities in urban Nanjing," Scientia Geographica Sinica, vol. 39, no. 3, pp. 433-441, 2019, in Chinese.

[46] P. Tesema Bulti, T. Besha Bedada, and L. Getahun Diriba, "Analyzing spatial distribution and accessibility of primary schools in Bishoftu Town Ethiopia," Spatial Information Research, vol. 27, no. 2, pp. 227-236, 2019.

[47] T. Zeng, Y. Deng, R. Yang, X. Zuo, Z. Chu, and X. Li, "Balanced development of compulsory education: cornerstone of education equity," Frontiers of Education in China, vol. 2, no. 4, pp. 469-493, 2007.

[48] H. Yang, X. Zhang, L. Li et al., "Changing spatial pattern and accessibility of primary and secondary schools in a poor mountainous country:a case study of Song County,Henan Province," Progress in Geography, vol. 37, no. 4, pp. 556-566, 2018, in Chinese.

[49] E. Talen, "School, community, and spatial equity: an empirical investigation of access to elementary schools in West Virginia," Annals of the Association of American Geographers, vol. 91, no. 3, pp. 465-486, 2001.

[50] M. Guo and B. Hu, "Assessing the spatial layout efficiency of basic educational resources by an improved coverage model: a case study of Nanchang City, China," Journal Of Geo-Information Science, vol. 21, no. 6, pp. 875-886, 2019, in Chinese.

[51] K. Liu and R. Liu, "Spatial concentration tendency and unbalanced development of elementary and middle schools under the background of urbanization in central rural area: a case study of Biyang county in Henan Province," Geographical Research, vol. 36, no. 9, pp. 1680-1696, 2017, in Chinease.

[52] S. Kučerová and Z. Kučera, "Changes in the spatial distribution of elementary schools and their impact on rural communities in Czechia in the second half of the 20th century," Journal of Research in Rural Education, vol. 27, no. 11, pp. 1-7, 2012. 